\title{
Reduction in Stages and Complete Quantization of the MIC-Kepler Problem
}

\author{
Ivaïlo M. Mladenov ${ }^{*}$ and Vasil V. Tsanov ${ }^{\dagger}$
}

4 May, 1998

*Institute of Biophysics, Bulgarian Academy of Sciences, Acad. G. Bonchev Str., Bl. 21, Sofia-1113, Bulgaria

${ }^{\dagger}$ Department of Mathematics, University of Sofia, 5 James Bourchier Bld., 1126 Sofia, Bulgaria

\begin{abstract}
The one-parameter deformation family of the standard Kepler problem known as the MIC-Kepler problem is completely quantized using the explicit momentum mapping of the torus actions on some toric manifolds and some equivariant cohomology theory. These manifolds appear as symplectic faces of the system. At any level of the reduction process the geometric quantization scheme produces all relevant quantum-mechanical numbers.
\end{abstract}

Key words: geometric quantization, equivariant cohomoligies

AMS subject classifications: 58F06, 58F07, 81S10

\footnotetext{
*Research partially supported by Bulgarian NSF-Project F-644/96.

${ }^{\dagger}$ Research partially supported DFGvproject number 436 BUL 113/96/5.
} 


\section{Introduction}

As a rule phase spaces of the most interesting classical Hamiltonian systems are cotangent bundle of smooth configuration manifolds and their quantization does not present serious problems (see Sect.2). However, the first step - the prequantization - produces only part of the quantum numbers and one should use other devices in order to obtain the spectrum of the complete set of Dirac observables. Here we present a detailed treatment of a concrete dynamical system and show that reduction in stages (either at classical or quantum level) produces the desired information about the spectrum. The system in question is the one-parameter deformation family of the Kepler problem known as MICKepler problem(see Sect.4). We should point out that despite geometric quantization concept is thirty years old such treatment is absent even for the standard Kepler problem. A possible explanation of this situation can be traced back to the general fact that one can quantize unambiguously only functions which are polynomials up to a second degree in phase space coordinates while the square of the angular momentum which is fourth degree polynomial does not belongs to this set. On the other side the choice of the momentum as an element of the complete set of observables is dictated by the spherical symmetry of the problem. Another wellknown fact is that the symmetries manifest themselves in the possibilities of separating the variables in the Schrödinger equation in different coordinate systems and this is connected with the existence of constants of motion. Simultaneous diagonalization of the Hamiltonian and the third components of momentum and RungeLenz vector corresponds to separation of variables in parabolic coordinates and has been noticed by Bargmann. Working in much more abstract setting we will follow essentially the same idea in order to derive missing quantum numbers. ¿From mathematical point of view the results which will be presented below follow from quantization of the momentum map associated with free torus actions on some symplectic (toric) manifolds.

\section{Geometric Quantization}

\subsection{Kostant-Souriau Programme}

On any symplectic manifold $(M, \omega)$ the symplectic form $\omega$ generates a Lie algebraic structure in the space $R^{\infty}(M)$ of smooth real-valued functions on $M$. The problem for finding the representations of $R^{\infty}(M)$ was approached for the first time by Dirac[1] in the case $\left(M \equiv R^{2 n}, \omega \equiv d p \wedge d q\right)$, and after that has been generalized by Segal[2] for the phase spaces which are cotangent bundles and finally by Kostant[3] and Souriau[4] for arbitrary symplectic manifolds. The starting point is the observation that if we are able to associate with every classical variable a quantum one then the commutator of two quantum variables should represents up to a multiplicative number the Poisson bracket of the classical ones. This part of the programme can be easily realized and nowadays is referred as prequantization. Below we summarize the relevant notion and definitions.

Definition 2.1 The symplectic manifold $(M, \omega)$ is pre-quantizable if $[\omega / 2 \pi]$ is in the image of the map 


$$
H_{\text {Cech }}^{2}(M, \mathbb{Z}) \rightarrow H_{\text {deRham }}^{2}(M, \mathbb{R}),
$$

where [ ] denotes the de Rham cohomological class.

When $M$ is a compact manifold this condition is equivalent to

$$
\frac{1}{2 \pi} \int_{\sigma} \omega \in \mathbb{Z}, \text { for every two }- \text { cycle } \sigma \in H_{2}(M, \mathbb{Z}) .
$$

and produces the quantization of charge, spin and energy levels of some physical systems. If $(M, \omega)$ is pre-quantizable, there exists a hermitian line bundle $L \rightarrow M$, whose Chern class is $\frac{1}{2 \pi}[\omega]$, equipped with a connection $\nabla$ which curvature form is $-i \omega$ and hermitian scalar product $h($,$) that is invariant with respect to the parallel transport [3,5,6,7]$. The irreducibility of the representations which is the second stage (quantization) of the programme is achieved by introducing additionally a new structure called polarization. A real polarization over $M$ is a such map that juxtapose to each point $m \in M$ a real subspace $F_{m} \subset T_{m}(M)$ which is maximally isotropic integrable distribution.

Example 2.2 Let $Q$ be a smooth manifold and let $T^{*} Q$ be its cotangent bundle. If $\left\{p_{i}, q_{i}\right\}$ are the local canonical coordinates in $T^{*} Q$, then an easy check shows that the vector fields

$$
X_{1}=\frac{\partial}{\partial p_{1}}, X_{2}=\frac{\partial}{\partial p_{2}}, \ldots, \quad X_{n}=\frac{\partial}{\partial p_{n}}
$$

define a real polarization over $T^{*} Q$ which is known as vertical polarization.

Example 2.3 The two-dimensional sphere does not allows real polarization because of the non-existence of non-singular real vector field on $S^{2}$.

This situation suggests also the generalization of the above notion, namely: A complex polarization over $M$ is a map $F$ which assigns to each point $m \in M$ a subspace $F_{m}$ of $T_{m}{ }^{C}(M)$ which is maximally isotropic integrable distribution, and besides the distribution $D_{m}=F_{m} \cap \bar{F}_{m}$ is of some fixed dimension $\kappa$ at each point $m \in M$. The polarization $F$ is called Kählerian if $F_{m} \cap \bar{F}_{m}=0$. For any kind of polarization $F$ the potential $\theta$ of the symplectic form $\omega$ (i.e. $\omega=d \theta$ ) is called an adapted to it if $\theta(X)=0$ for every $X \in F$. The quantum pre-Hilbert space is built up by the polarized sections of $L$ which definition is as follows: Let $M, \omega, L, \nabla$ and $F$ be as defined above. The polarized sections of $L$ form the line bundle

$$
L^{F}=\left\{s \in \operatorname{Sect}(\mathrm{L}) ; \nabla_{X} s=0, \quad \text { for all } X \in \mathfrak{X}(M, F)\right\}
$$

In order to have true Hilbert space we need some measure(or density) which is an element of a second line bundle. This can be introduced if we consider the elements of the cotangent bundle $T^{*}(M)$ that vanish on $F$ and form a subbundle $F^{\circ} \subset T_{C}^{*}(M)$ which is called annihilator of $F$. By the very definition of the symplectic form we have that the map

$$
v \in F \rightarrow i(v) \omega \in F^{\circ}
$$


is an isomorphism of $F$ and $F^{\circ}$. This means that we can form the line bundle $K_{F}=\wedge^{n} F^{\circ}$ over $M$ that will be further referred as a canonical bundle of $F$. If $\left\{v_{1}, v_{2}, \ldots, v_{n}\right\}$ is a basis of $F$, then

$$
K_{\omega}=i\left(v_{1}\right) \omega \wedge i\left(v_{2}\right) \omega \wedge \ldots \wedge i\left(v_{n}\right) \omega
$$

is a basis in $K_{F}$ and for every $g \in G L(n, C),\left(K^{g}\right)_{\omega}=\operatorname{detg} . K_{\omega}$.

Let $(M, \omega)$ be a symplectic manifold and $F$ is a complex polarization on it. We will say that $M$ is a metaplectic manifold if there exists a line bundle $N^{1 / 2}$ over $M$ such that

$$
N^{1 / 2} \otimes N^{1 / 2}=K_{F}
$$

One can show that $(M, \omega)$ is metaplectic if and only if the first Chern class of $K_{F}$ is zero modulo two and this property does not depend on the choice of $F$. In this case the group $H^{1}\left(M, \mathbb{Z}_{2}\right)$ parameterizes the set of "square roots", i.e. the set of all $N^{1 / 2}$ which

satisfy the above condition. The sections of $N_{F}^{1 / 2}$ which are constant along $F$ are called half-forms normal to $F$.

The line bundle $\tilde{Q}=L^{F} \otimes N_{F}^{1 / 2}$ over $M$ is called a quantum line bundle because its sections are considered as elements of the Hilbert space $\mathcal{H}^{F}$. The classical observables which can be quantized directly are those that preserve the polarization $F$, i.e. $\{f \in$ $\left.R^{\infty}(M) ;\left[X_{f}, F\right] \subset F\right\}$, where $X_{f}$ is defined by the equation $i\left(X_{f}\right) \omega=-d f$. If $\psi=s \otimes \nu$, where $\psi \in \Gamma(\tilde{Q}), \quad s \in \Gamma\left(L^{F}\right), \nu \in \Gamma\left(N_{F}^{1 / 2}\right)$ are sections of the corresponding line bundles, the associated with $f$ quantum operator acts in $\mathcal{H}^{F}$ as specified below :

$$
\hat{f}(\psi)=\left(-i \nabla_{X_{f}}+f\right) s \otimes \nu-i s \otimes \mathcal{L}\left(X_{f}\right) \nu
$$

Identifying the sections of $L^{F}$ with functions on $M$ (which is possible because $L^{F}$ is a line bundle) the action of $\hat{f}$ in $\mathcal{H}^{F}$ can be written in the form

$$
\hat{f} \psi=\left(-i X_{f}-\theta\left(X_{f}\right)+f\right) \varphi \otimes \nu-i \varphi \otimes \mathcal{L}\left(X_{f}\right) \nu
$$

where $\theta$ is the potential one form of $\omega$.

Actually this explicit formula has found very few applications (cf. Sect.6) as most of the considerations end with checking the consistency of the scheme relying on (2.3).

\subsection{Czyz-Hess Scheme}

After cotangent bundles and co-adjoint orbits Kählerian manifolds form another important class of symplectic manifolds. According Darboux theorem all symplectic manifolds (of fixed dimension) are locally the same but in practice they appear with some additional geometric structure. It presence in the setting of geometric quantization helps in many cases to answer definitely the question if the given symplectic manifold $(M, \omega)$ allows such quantization. A trivial example is provided by even-dimensional complex projective spaces. The well-known fact for these manifolds is that

$$
H^{2}\left(C P^{2 n}, \mathbb{Z}_{2}\right) \cong \mathbb{Z}_{2}
$$


On the other hand we know that the symplectic manifold $(M, \omega)$ can be quantized if $M$ is a metaplectic manifold, i.e. $H^{2}\left(M, \mathbb{Z}_{2}\right)=0$. So, even-dimensional complex projective spaces can not be treated in Kostant-Souriau scheme. On the other hand they appear as orbit manifolds of the odd-dimensional harmonic oscillators which form an important class of dynamical systems. Fortunately this problem can be taken away by slight modification of geometric quantization scheme as developed by Czyz[8] and Hess[9] and outlined bellow.

Definition 2.4 Let $(M, \omega)$ be such Kählerian manifold that $[q]=\frac{1}{2 \pi}[\omega]-\frac{1}{2} c_{1}(M)$ belongs to the image of $\epsilon: H^{2}(M, \mathbb{Z}) \rightarrow H^{2}(M, \mathbb{R})$ and $q$ is positive, i.e. $q(\sigma) \geq 0$ for any positively oriented two-cycle $\sigma \in \mathrm{H}_{2}(M, \mathbb{R})$. The complex line bundle $Q$ whose first Chern class $c_{1}(Q)$ is $q$ is called quantum bundle.

If the bundles $L^{F}$ and $N_{F}^{1 / 2}$ exist then there exists also the bundle $\tilde{Q}=L^{F} \otimes N_{F}^{1 / 2}$ so that $c_{1}(\tilde{Q})=c_{1}(Q)$ and therefore $\tilde{Q}$ and $Q$ are equivalent. Among symplectic manifolds the Kählerian ones are those which possess canonical anti-holomorphic polarization that makes identification of quantum states with holomorphic sections quite natural. Now, fixing a positive harmonic representative $\eta \in c_{1}(Q)$ and connection $\nabla$ which curvature is $-2 \pi i \eta$ we are in position to define also and $\nabla$ - invariant hermitian structure $h_{\eta}($,$) on$ $Q$. We recall that, the curvature of the hermitean metric $h_{\eta}$ on the bundle $Q$ satisfies

$$
\frac{i}{2 \pi} \partial \bar{\partial} \log h_{\eta} \simeq \frac{\omega}{2 \pi}-\frac{1}{2} c_{1}(M)
$$

The space of holomorphic sections $H^{0}((M, Q)$ of $Q$ can be converted into Hilbertian space $\mathcal{H}$ if we introduce the scalar product

$$
<s, t>=\int_{M} h_{\eta}(s, t) \Omega_{\eta}, \quad \omega_{\eta}=2 \pi \eta, \quad s, t \in \Gamma(M, Q), \quad n=\frac{1}{2} \operatorname{dim} M .
$$

and where $\Omega_{\eta}:=\frac{(-1)^{n(n-1) / 2}}{n !} \omega_{\eta} \wedge \omega_{\eta} \wedge \ldots \wedge \omega_{\eta}$ is the natural volume form on $M$. If our manifold $M$ is simply-connected the hermitian structure is defined up to a positive factor and $\mathcal{H}$ is defined up to an isomorphism which depends on the choice of the connection $\nabla$. The representations are build up following the prequantization recipe in which $(L, \omega)$ is exchanged for $\left(Q, \omega_{\eta}\right)$ i.e. to the classical observable (i.e. a function $f$ on the phase space), there corresponds a quantum operator

$$
\delta(f) \in \text { End } H^{0}(M, Q) ; \quad \delta(f) s \equiv\left(-i \nabla_{X_{f}}+f\right) s
$$

where $s \in H^{0}(M, Q)$, and now the vector field $X_{f}$ is defined by:

$$
i\left(X_{f}\right) \omega_{\eta}=-d f .
$$

The only problem here is that $\omega_{\eta}$ is not always non-degenerated. More detailed exposition can be found in Czyz[8] and Hess[9]. 


\section{Classical and Quantum Reductions}

When a Lie group $G$ acts symplectically(canonically) on the phase space $(P, \omega)$ of the Hamiltonian system $(P, \omega, H)$ leaving the Hamiltonian $H$ invariant it generates quite naturally a mapping from $P$ into the dual space $\mathfrak{g}^{*}$ of its Lie algebra $\mathfrak{g}$ whose components are integrals of motion for the dynamical system. This means that the motion takes place inside a constraint submanifold $C \subset P$ and sometimes possesses gauge degrees of freedom. Passing on a new manifold where they are discarded is known for centuries in mechanics as reduction procedure and its modern formulation given below is due to Marsden and Weinstein [10].

Theorem 3.1 Let $(P, \omega)$ be a symplectic manifold on which acts canonically the Lie group $G$, and $J: P \rightarrow \mathfrak{g}^{*}$ be the Ad ${ }^{*}$-equivariant momentum mapping of this action. Let us suppose that $\mu \in \mathfrak{g}^{*}$ is a regular value of $J$ and that the isotropy group $G_{\mu}$ act freely and properly on $J^{-1}(\mu)$. Then $P_{\mu}=J^{-1}(\mu) / G_{\mu}$ is a symplectic manifold with a symplectic form defined by $\pi_{\mu}^{*} \omega_{\mu}=i_{\mu}^{*} \omega$ where $\pi_{\mu}: J^{-1}(\mu) \rightarrow P_{\mu}$ is the canonical projection and $i_{\mu}: P_{\mu} \rightarrow P$ is the embedding. Let $H: P \rightarrow \mathbb{R}$ is $G$-invariant Hamiltonian function. The flow induced on $P_{\mu}$ is also a Hamiltonian one with Hamiltonian function $H_{\mu}$ defined by the relation $H_{\mu} \circ \pi_{\mu}=H \circ i_{\mu}$.

If the Hamiltonian system $(M, \omega, H)$ allows a symmetry group action commuting with that of $G$, the reduced system $\left(P_{\mu}, \omega_{\mu}, H_{\mu}\right)$ keeps this symmetry.

A special case of the above theorem which will be of immediate interest in the sequel is the case when $P$ is a cotangent bundle $T^{*}(M)$ of some manifold $M$ on which acts freely and properly the one-parameter Lie group $G$. Let $M \rightarrow N=M / G$ be the induced principal $G$-bundle and $\tilde{\alpha}$ be the connection one-form. The reduced symplectic manifold $P_{\mu}$ is symplectomorphic with $T^{*} N$ which symplectic form $\omega_{\mu}$ is the sum of the canonical form on $T^{*} N$ and a magnetic term $\mu \tau_{N}^{*} d \tilde{\alpha}$ where $\tau_{N}$ is the canonical projection $\tau_{N}: T^{*} N \rightarrow N$ [11].

Thus, each Hamiltonian system with symmetry can be treated as dynamical system either on $(P, \omega)$ or $\left(P_{\mu}, \omega_{\mu}\right)$ and what is more important - there is no formal distinction at classical level between working on the initial or reduced phase space. There are plenty of strong results concerning the quantum mechanical counterpart of this situation which tell us when quantization and reduction are coherent procedures (see $[13,12,14,15])$. In order to give the reader the flavor what to expect in this situation and because we will make use of it we quote the following result:

Theorem 3.2 (Guillemin 6 Sternberg[13]). Let us suppose that the (extended) phase space $(P, \omega)$ is a compact and quantizable, $G$ is a compact Lie group, $0 \in \mathfrak{g}^{*}$ is a regular value of $J$ and $F$ is a Kählerian $G$-invariant polarization over $P$. Then, there exists an isomorphism between the $G$-invariant sections of $L^{F}$ and the sections of the quantum line bundle over the reduced phase space $\left(P_{0}, \omega_{0}\right)$.

The situation is even more favorite - in the above setting the reduction and (pre)- quantization are interchangeable procedures. 


\section{The MIC-Kepler Problem}

The Hamiltonian system $\left(T^{*} \dot{R}^{3}, \Omega_{\mu}, H_{\mu}\right)$, where

$$
\begin{gathered}
T^{*} \dot{R}^{3} \equiv T^{*}\left(R^{3} \backslash\{0\}\right) \equiv\left\{(p, q) \in R^{3} \times R^{3} ; q \neq 0\right\} \\
\Omega_{\mu}=d \theta+\sigma_{\mu}, \theta=\sum_{j=1}^{3} p_{j} d q_{j}, \sigma_{\mu}=-\mu /\left(2|q|^{3}\right) \sum_{i, j, k=1}^{3} \epsilon_{i j k} q_{i} d q_{j} \wedge d q_{k}, \\
H_{\mu}=\frac{1}{2}|p|^{2}-\alpha / r+\mu^{2} / 2 r^{2},|q|^{2}=q_{1}^{2}+q_{2}^{2}+q_{3}^{2}=r^{2}, \quad \alpha, \mu \in R, \alpha>0,
\end{gathered}
$$

is known as the MIC-Kepler problem[16, 17]. Using more or less standard physical terminology, the problem consists in studying the motion of charged particle in a field which is a superposition of a magnetic monopole field $\vec{B}_{\mu}=-\mu \vec{q} /|q|^{3}$ and the fields generated by the Newtonian potential $-\alpha / r$ and centrifugal potential $\mu^{2} / 2 r^{2}$. We will see that the energy level submanifolds $H_{\mu}^{-1}(E)$ for negative values of the energy are filled up with closed orbits. This hints a presence of "hidden" symmetry and "accidental" degeneracy of the spectrum. Actually, the "hidden" symmetry of the Hamiltonian system $\left(T^{*} \dot{R}^{3}, \Omega_{\mu}, H_{\mu}\right)$ is $S O(4)$ generated by the constants of motion

$$
\vec{L}^{\mu}=\vec{q} \times \vec{p}+\mu \vec{q} / r, \quad \vec{A}^{\mu}=\left(\vec{L}^{\mu} \times \vec{p}+\alpha \vec{q} / r\right) / \sqrt{-H_{\mu}}
$$

which have interpretation of a "total angular momentum" and generalized Runge-Lenz vector. The names are borrowed by the standard Kepler problem which can be viewed as a special "point" of this one-parameter deformation family. The classical Kepler problem $(\mu=0)$ was geometrically quantized by Simms[18] and Mladenov \& Tsanov[19]. Here we will apply the geometric quantization to extended and the reduced phase spaces of the Hamiltonian system $\left(T^{*} \dot{R}^{3}, \Omega_{\mu}, H_{\mu}\right)$ which results in coinciding spectra. We will present them as

Theorem 4.1 (Mladenov\& Tsanov[17]). The discrete spectrum (bound states) of the $M I C-K e p l e r$ problem ( $\alpha$ - fixed, $\mu$-fixed and quantized) consists of energy levels:

$$
E_{N}=-\alpha^{2} / 2 N^{2}, \quad N=|\mu|+1,|\mu|+2,|\mu|+3 \ldots
$$

The magnetic charge $\mu$ can take the values

$$
\mu=0, \pm \frac{1}{2}, \pm 1, \pm \frac{3}{2}, \pm 2 \ldots
$$

and the multiplicity of the energy level $E_{N}$ is:

$$
m\left(E_{N}\right)=N^{2}-\mu^{2} .
$$




\section{Conformal Kepler Problem}

The Hamiltonian system $\left(T^{*} \dot{R}^{4}, \Omega, H_{\alpha}\right)$, where :

$$
\begin{gathered}
T^{*} \dot{R}^{4}=T^{*}\left(R^{4} \backslash\{0\}\right)=\left\{(y, x) \in R^{4} \times R^{4} ; x \neq 0\right\}, \\
\Omega=d y \wedge d x=\sum_{j=1}^{4} d y_{j} \wedge d x_{j}
\end{gathered}
$$

and

$$
H_{\alpha}=\left(|y|^{2}-8 \alpha\right) / 8|x|^{2}, \alpha-\text { fixed constant }
$$

is known as a conformal Kepler problem [20]. Let us introduce additionally two other Hamiltonian functions on the phase space $\left(T^{*} \dot{R}^{4}, \Omega\right)$, namely, that one of the Harmonic oscillator :

$$
K_{\lambda}=\left(|y|^{2}+\lambda^{2}|x|^{2}\right) / 2, \quad \lambda-\text { an arbitrary positive constant, }
$$

and that one of the "momentum":

$$
M=\frac{1}{2}\left(x_{1} y_{2}-x_{2} y_{1}+x_{3} y_{4}-x_{4} y_{3}\right) .
$$

Lemma 5.1 Let $E<0$ and $\lambda=\sqrt{-8 E}$. Then

$$
H_{\alpha}^{-1}(E)=K_{\lambda}^{-1}(4 \alpha)
$$

and the flows defined by the Hamiltonians $H_{\alpha}$ and $K_{\lambda}$ coincide on these hypersurfaces up to re-parameterization.

Proof. Taking into account the above definition it is obvious that we have

$$
4|x|^{2}\left(H_{\alpha}+\lambda^{2} / 8\right)=K_{\lambda}-4 \alpha,
$$

which proves the first statement. Further on $H_{\alpha}$ and $K_{\lambda}$ will be denoted by $H$ and $K$. In order to prove the second one we need only to notice that the Hamiltonian vector fields $X_{H}$ and $X_{K}$ when restricted to energy level submanifolds $H^{-1}(E)=K^{-1}(4 \alpha)$ are related as follows :

$$
4|x|^{2} X_{H}=X_{K},
$$

and so the proof of the lemma is complete.

The complex coordinates on $\left(T^{*} \dot{R}^{4}, \Omega\right)$ written below depend on the same arbitrary positive constant $\lambda$ chosen above

$$
\begin{aligned}
& z_{1}=\lambda\left(x_{1}+i x_{2}\right)-i\left(y_{1}+i y_{2}\right), \quad z_{2}=\lambda\left(x_{3}+i x_{4}\right)-i\left(y_{3}+i y_{4}\right), \\
& z_{3}=\lambda\left(x_{1}-i x_{2}\right)-i\left(y_{1}-i y_{2}\right), \quad z_{4}=\lambda\left(x_{3}-i x_{4}\right)-i\left(y_{3}-i y_{4}\right) .
\end{aligned}
$$


In these coordinates $T^{*} \dot{R}^{4} \equiv \mathbb{C}^{4} \backslash D$, where

$$
D=\left\{z \in \mathbb{C}^{4}, z_{1}=-\bar{z}_{3}, z_{2}=-\bar{z}_{4}\right\}
$$

and the symplectic form $\Omega$ turns out to be (up to a multiplicative constant) the standard Kähler form on $\mathbb{C}^{4}$

$$
\Omega=\frac{i}{4 \lambda} d z \wedge d \bar{z}=\frac{i}{4 \lambda} \sum_{j=1}^{4} d z_{j} \wedge d \bar{z}_{j} .
$$

Finally, the hamiltonian functions $K$ and $M$ can be written in these coordinates as

$$
K=\left(\left|z_{1}\right|^{2}+\left|z_{2}\right|^{2}+\left|z_{3}\right|^{2}+\left|z_{4}\right|^{2}\right) / 4
$$

and

$$
M=\left(\left|z_{1}\right|^{2}+\left|z_{2}\right|^{2}-\left|z_{3}\right|^{2}-\left|z_{4}\right|^{2}\right) / 8 \lambda
$$

It should be noted that these hamiltonians and the symplectic form $\Omega$ are well defined over the manifold

$$
\dot{\mathbb{C}}^{4}=\mathbb{C}^{4} \backslash\{0\} \supset T^{*} \dot{R}^{4} .
$$

Let $K_{t}, M_{t}$ denote the flows of the Hamiltonian systems $\left(\dot{\mathbb{C}}^{4}, \Omega, K\right)$, $\left(\dot{\mathbb{C}}^{4}, \Omega, M\right)$.

Lemma 5.2 For every $z \in \dot{\mathbb{C}}^{4}$ and $s, t \in R$, the corresponding flows are:

$$
\begin{aligned}
& K_{t} z=\left(e^{i \lambda t} z_{1}, e^{i \lambda t} z_{2}, e^{i \lambda t} z_{3}, e^{i \lambda t} z_{4}\right), \\
& M_{s} z=\left(e^{i s / 2} z_{1}, e^{i s / 2} z_{2}, e^{-i s / 2} z_{3}, e^{-i s / 2} z_{4}\right) .
\end{aligned}
$$

In particular, the flows of all three Hamiltonians $H, K$ and $M$ commute where defined. Proof. The explicit expressions for the flows $K_{t}, M_{s}$ are obtained by direct calculations. The last assertion follows from these expressions and Lemma 5.1.

In view of the lemma that have been just proved, the flow $M_{s}$ defines a symplectic $U(1)$ action over $\dot{\mathbb{C}}^{4}$. The "momentum" for this action is $M$ itself. Let us remark that the set $D$ and consequently its complementary set $T^{*} \dot{R}^{4}$ are invariant under this $U(1)$-action. Through every point there pass just one orbit and the Hamiltonian function $H$ invariant on these orbits. All this means that the Hamiltonian system $\left(T^{*} \dot{R}^{4}, \Omega, H\right)$ can be reduced with respect to $U(1)$. The result of this reduction is summarized in the following lemma:

Lemma 5.3 [20, 17] Let $\mu \in R$ be the value of the momentum map of the lifted Hopf action on $T^{*} \dot{R}^{3}$. Then

$$
M^{-1}(\mu) / U(1) \equiv T^{*} \dot{R}^{3}
$$

and when reduced $\Omega$ and $H$ produce $\Omega_{\mu}$ and $H_{\mu}$, i.e. one ends with the $M I C-K e p l e r$ problem. 
Besides, if one reduce the constants of motion of the conformal Kepler problem:

$$
\begin{array}{ll}
M_{1}=\left(z_{1} \bar{z}_{2}+z_{2} \bar{z}_{1}-z_{3} \bar{z}_{4}-z_{4} \bar{z}_{3}\right) / 8 \lambda & A_{1}=\left(z_{1} \bar{z}_{2}+z_{2} \bar{z}_{1}+z_{3} \bar{z}_{4}+z_{4} \bar{z}_{3}\right) / 8 \lambda \\
M_{2}=\left(z_{1} \bar{z}_{2}-z_{2} \bar{z}_{1}+z_{3} \bar{z}_{4}-z_{4} \bar{z}_{3}\right) / 8 \lambda i & A_{2}=\left(z_{1} \bar{z}_{2}-z_{2} \bar{z}_{1}-z_{3} \bar{z}_{4}+z_{4} \bar{z}_{3}\right) / 8 \lambda i \\
M_{3}=\left(\left|z_{1}\right|^{2}-\left|z_{2}\right|^{2}-\left|z_{3}\right|^{2}+\left|z_{4}\right|^{2}\right) / 8 \lambda & A_{3}=\left(\left|z_{1}\right|^{2}-\left|z_{2}\right|^{2}+\left|z_{3}\right|^{2}-\left|z_{4}\right|^{2}\right) / 8 \lambda
\end{array}
$$

one gets the momentum $\vec{L}^{\mu}$ and the generalized Runge-Lenz vector $\vec{A}^{\mu}$ which are constants of the motion for the MIC-Kepler problem.

\section{Quantization of the Extended Phase Space}

Definition 6.1 The level hypersurfaces of the map $J: \mathbb{C}^{4} \backslash D \rightarrow R^{2}, J(z)=(K(z), M(z))$ are called energy-momentum manifolds.

$$
\mathcal{E} \mathcal{M}(\lambda, \mu)=\left\{(y, x) \in T^{*} \dot{R}^{4} ; K=4 \alpha, \quad M=\mu\right\} .
$$

Under reduction $\mathcal{E} \mathcal{M}(\lambda, \mu)$ falls down $\left(\right.$ via $\pi_{\mu}$ ) over the energy hypersurface $H_{\mu}=-\lambda^{2} / 8$ $(\lambda=\sqrt{-8 E})$ of the MIC-Kepler problem. As a set $\mathcal{E} \mathcal{M}(\lambda, \mu)$ is not empty if $\lambda$ and $\mu$ satisfy the condition

$$
\lambda|\mu| \leq 2 \alpha
$$

In this section we suppose (by the reasons that will be clarified in the next one) that we have strong inequality $\lambda|\mu|<2 \alpha$. Following [21] (see also [22]-[23]) we will change our viewpoint and will consider $\left(T^{*} \dot{R}^{4}, \Omega\right)$ as an "extension" of $\left(T^{*} \dot{R}^{3}, \Omega_{\mu}\right)$.

We will prove Theorem 4.1 working with the complex coordinates defined in $(5.2)$, the polarization $F$ "spanned" by the anti-holomorphic directions $\left\{\frac{\partial}{\partial \bar{z}_{1}}, \frac{\partial}{\partial \bar{z}_{2}}, \frac{\partial}{\partial \bar{z}_{3}}, \frac{\partial}{\partial \bar{z}_{4}}\right\}$ and adapted potential $\theta=-\frac{i}{4 \lambda} \bar{z} d z$ of $\Omega$. The Hilbert space consists of "wave functions" of the form $\psi=\varphi \otimes \nu$ where $\varphi$ is holomorphic and

$$
\nu=\left(d z_{1} \wedge d z_{2} \wedge d z_{3} \wedge d z_{4}\right)^{1 / 2} .
$$

Essentially Dirac's idea concerning quantization in the presence of constraints that are not eliminated at the classical level is that they should be enforced at the quantum one. In our case the constraints $K=4 \alpha$ and $M=\mu$ select the energy-momentum manifold $\mathcal{E} \mathcal{M}(\lambda, \mu)$ and therefore the acceptable quantum states are those that belong to the subspace $\mathcal{H}_{J}$ of $\mathcal{H}$ defined below:

$$
\mathcal{H}_{J}=\{\psi \in \mathcal{H} ; \hat{K} \psi=4 \alpha \psi, \hat{M} \psi=\mu \psi\} .
$$

Taking into account all of the above and formula (2.4) we write down the quantized version of our operators as

$$
\begin{aligned}
\hat{K} \psi & =\lambda\left(z_{1} \frac{\partial}{\partial z_{1}}+z_{2} \frac{\partial}{\partial z_{2}}+z_{3} \frac{\partial}{\partial z_{3}}+z_{4} \frac{\partial}{\partial z_{4}}+2\right) \varphi \otimes \nu \\
& =\lambda(\mathcal{N}+2) \psi=4 \alpha \psi, \quad \mathcal{N}=0,1,2, \ldots
\end{aligned}
$$


and

$$
\hat{M} \psi=\frac{1}{2}\left(z_{1} \frac{\partial}{\partial z_{1}}+z_{2} \frac{\partial}{\partial z_{2}}-z_{3} \frac{\partial}{\partial z_{3}}-z_{4} \frac{\partial}{\partial z_{4}}\right) \varphi \otimes \nu=\mu \psi,
$$

where $\varphi$ is a homogeneous monom of degree $\mathcal{N}$ in $z_{1}, z_{2}, z_{3}$ and $z_{4}$.

Introducing $N=\mathcal{N} / 2+1$ and solving

$$
2 N \sqrt{-8 E}=4 \alpha
$$

we obtain the energy spectrum $E_{N}=-\alpha^{2} / 2 N^{2}$ as well

$$
\begin{aligned}
& n_{1}+n_{2}+n_{3}+n_{4}=2 N-2, \\
& n_{1}+n_{2}-n_{3}-n_{4}=2 \mu, \quad n_{i} \geq 0, i=1,2,3,4 .
\end{aligned}
$$

The last constraint relation is equivalent to Dirac's quantization of the magnetic charge:

$$
\mu=0, \pm \frac{1}{2}, \pm 1, \pm \frac{3}{2}, \pm 2 \ldots
$$

Besides we get :

$$
n_{1}+n_{2}=N+\mu-1=\mathcal{N}_{1}=0,1,2, \ldots
$$

and

$$
n_{3}+n_{4}=N-\mu-1=\mathcal{N}_{2}=0,1,2, \ldots
$$

which combined tell us that the possible values of $N$ are given by the formula $N=|\mu|+1$, $|\mu|+2,|\mu|+3, \ldots$

In order to find the degeneracies $m\left(E_{N}\right)$ one should notice that $\varphi$ can be represented as a product $\varphi_{1}\left(z_{1}, z_{2}\right) \cdot \varphi_{2}\left(z_{3}, z_{4}\right)$ of homogeneous monomials of degree $\mathcal{N}_{1}$ and $\mathcal{N}_{2}$ respectively. So, the dimension of the Hilbert space $\mathcal{H}_{\mu, N}$ is :

$$
m\left(E_{N}\right)=\operatorname{dim} \mathcal{H}_{\mu, N}=\left(\mathcal{N}_{1}+1\right)\left(\mathcal{N}_{2}+1\right)=N^{2}-\mu^{2},
$$

and this ends the proof of the theorem.

Remark 6.2 The Hilbert space $\mathcal{H}_{\mu, N}$ is the carrier space for the unitary irreducible representation $\left(\frac{\mathcal{N}_{1}}{2}, \frac{\mathcal{N}_{2}}{2}\right)=\left(\frac{N+\mu-1}{2}, \frac{N-\mu-1}{2}\right)$ of the global symmetry group of the MIC-Kepler problem $\operatorname{Spin}(4) \cong S U(2) \otimes S U(2)$ ( $\mu$-half-integer) or $S O(4)$ ( $\mu$-integer $)$.

The wave functions in $\mathcal{H}=\oplus \mathcal{H}_{\mu, N}$ are labeled uniquely by four quantum numbers which are the eigenvalues of the complete set of commuting operators $\hat{M}(\mu), \hat{H}(N), \hat{M}_{3}(m), \hat{A}_{3}(\ell)$, where

$$
\hat{M}_{3} \psi=\frac{1}{2}\left(z_{1} \frac{\partial}{\partial z_{1}}-z_{2} \frac{\partial}{\partial z_{2}}-z_{3} \frac{\partial}{\partial z_{3}}+z_{4} \frac{\partial}{\partial z_{4}}\right) \psi=m \psi
$$

and

$$
\hat{A}_{3} \psi=\frac{1}{2}\left(z_{1} \frac{\partial}{\partial z_{1}}-z_{2} \frac{\partial}{\partial z_{2}}+z_{3} \frac{\partial}{\partial z_{3}}-z_{4} \frac{\partial}{\partial z_{4}}\right) \psi=\ell \psi
$$


Looking at (6.1) and (6.2) one can conclude immediately that $m$ and $l$ can take either integer or half-integer values. It is to be noted also that this result is closely related with convexity theorem of the torus actions on symplectic manifolds [23]. Indeed, let us consider the flows $U_{\varsigma}, V_{\tau}$ generated by $M_{3}$ and $A_{3}$,

$$
\begin{aligned}
& U_{\varsigma} z=\left(e^{i \varsigma / 2} z_{1}, e^{-i \varsigma / 2} z_{2}, e^{-i \varsigma / 2} z_{3}, e^{i \varsigma / 2} z_{4}\right) \\
& V_{\tau} z=\left(e^{i \tau / 2} z_{1}, e^{-i \tau / 2} z_{2}, e^{i \tau / 2} z_{3}, e^{-i \tau / 2} z_{4}\right)
\end{aligned}
$$

in conjunction with $K_{t}$ and $M_{s}$. Doing so we realize that we have at disposition an action of the four-torus $T^{4}$ on our symplectic manifold $\left(\dot{\mathbb{C}}^{4}, \Omega\right)$. Introducing new "time" variables

$$
\begin{aligned}
& \phi_{1}=\lambda t+\frac{s}{2}+\frac{\varsigma}{2}+\frac{\tau}{2} \\
& \phi_{2}=\lambda t+\frac{s}{2}-\frac{\varsigma}{2}-\frac{\tau}{2} \\
& \phi_{3}=\lambda t-\frac{s}{2}-\frac{\varsigma}{2}+\frac{\tau}{2} \\
& \phi_{4}=\lambda t-\frac{s}{2}+\frac{\varsigma}{2}-\frac{\tau}{2}
\end{aligned}
$$

this action takes the form

$$
\Phi(\phi, z)=\left(e^{i \phi_{1}} z_{1}, e^{i \phi_{2}} z_{2}, e^{i \phi_{3}} z_{3}, e^{i \phi_{4}} z_{4}\right)
$$

and the associated moment $J_{\Phi}$ is readily given by

$$
J_{\Phi}(z)=\frac{1}{8 \lambda}\left(\left|z_{1}\right|^{2},\left|z_{2}\right|^{2},\left|z_{3}\right|^{2},\left|z_{4}\right|^{2}\right)
$$

which makes obvious that the image set is convex. Besides, our representation space is spanned by the homogeneous polynomials of degree $\mathcal{N}$ in the variables $z_{1}, z_{2}, z_{3}, z_{4}$ on which the torus element $g=\left(e^{i \phi_{1}}, e^{i \phi_{2}}, e^{i \phi_{3}}, e^{i \phi_{4}}\right)$ is represented by the transformation

$$
\sum a_{n} z^{n} \rightarrow \sum a_{n} e^{i \phi . n} z^{n}
$$

The multi-indices $n=\left(n_{1}, n_{2}, n_{3}, n_{4}\right)$ which appear above obey $n_{1}+n_{2}+n_{3}+n_{4}=\mathcal{N}$ and provide labels for the irreducible multiplicity-free representations $\rho_{\mathcal{N}}$ of the torus $T^{4}$.

\section{Quantization of the Orbit Manifolds}

In Section 5 we have established that the energy level submanifolds consist entirely of closed orbits. This allows $H_{\mu}^{-1}(E)$ to be factorized with respect to dynamical flow and the so obtained manifold $H_{\mu}^{-1}(E) / U(1)=\mathcal{O}_{\mu}(E)$ is known as an orbit manifold. Its complete description as a symplectic manifold is given below: 
Theorem 7.1 (Mladenov $\&$ Tsanov[17]). Let $E<0$ and $\lambda=\sqrt{-8 E}$. Then :

$$
\begin{array}{rll}
\text { i) } \quad \text { if } \lambda|\mu|<2 \alpha & \mathcal{O}_{\mu}(E) \cong P^{1} \times P^{1} \\
\text { ii) } \quad \text { if } \lambda|\mu|=2 \alpha & \mathcal{O}_{\mu}(E) \cong P^{1} \\
\text { iii) } \quad \text { if } \lambda|\mu|>2 \alpha & H_{\mu}^{-1}(E) \equiv \emptyset
\end{array}
$$

The reduced symplectic form over $P^{1} \times P^{1}$ is :

$$
\Omega_{\mu}(E)=\frac{2 \pi(2 \alpha+\lambda \mu)}{\lambda} \omega_{1}+\frac{2 \pi(2 \alpha-\lambda \mu)}{\lambda} \omega_{2}
$$

where

$$
\omega_{j}=\frac{i}{2 \pi} \frac{d \zeta_{j} \wedge d \bar{\zeta}_{j}}{\left(1+|\zeta|^{2}\right)^{2}}, \quad j=1,2
$$

and $\left(\zeta_{1}, \zeta_{2}\right)$ are whichever non-homogeneous coordinates on $P^{1} \times P^{1}$.

The symplectic form over $P^{1}$ in item $\left.i i\right)$ is the respective non-zero component of $\mathcal{O}_{\mu}(E)$ (depending on the sign of $\mu$ ). This theorem reduces quantization of the MIC-Kepler problem to geometric quantization of the compact Kähler manifolds $P^{1} \times P^{1}$ and $P^{1}$. The proof is based on the following lemma:

Lemma $7.2 \mathcal{O}_{\mu}(E) \cong J^{-1}(4 \alpha, \mu) / U(1) \times U(1)$.

Proof. When $\mu \neq 0$ the orbits of the Hamiltonian $H$ coincides with that of $K$ described by Lemma 5.1. In particular, none of them belongs to $\dot{\mathbb{C}}^{4} \backslash T^{*} \dot{R}^{4}=D \backslash\{0\}$ and therefore we have one-to-one correspondence between the orbits of the MIC-Kepler problem on the energy hypersurface $H_{\mu}=E$ and the orbits of the torus action on $J^{-1}(4 \alpha, \mu)$ described in Lemma 5.2 and this implies that the orbit spaces are identical.

What remains to be done in order to prove Theorem 7.1 is to describe properly $J^{-1}(4 \alpha, \mu)$. For that purpose we remark that the system of equations $K=4 \alpha, M=\mu$ is equivalent to the system

$$
\left|z_{1}\right|^{2}+\left|z_{2}\right|^{2}=4(2 \alpha+\lambda \mu), \quad\left|z_{3}\right|^{2}+\left|z_{4}\right|^{2}=4(2 \alpha-\lambda \mu)
$$

so we can conclude that

$$
J^{-1}(4 \alpha, \mu)= \begin{cases}S^{3} \times S^{3} & \text { when } \lambda|\mu|<2 \alpha \\ S^{3} & \text { when } \lambda|\mu|=2 \alpha \\ \emptyset & \text { when } \lambda|\mu|>2 \alpha\end{cases}
$$

The projection $p: S^{3} \times S^{3} \rightarrow P^{1} \times P^{1}$ is defined through the Hopf's map of the corresponding factors

$$
p\left(z_{1}, z_{2}, z_{3}, z_{4}\right)=\left(\left[z_{1}: z_{2}\right],\left[z_{3}: z_{4}\right]\right),
$$

where $\left[z_{1}: z_{2}\right],\left[z_{3}: z_{4}\right]$ are the homogeneous coordinates over $P^{1} \times P^{1}$. In accordance with Lemma 7.2 the projection $p$ is just the factor-map

$$
J^{-1}(4 \alpha, \mu) \rightarrow J^{-1}(4 \alpha, \mu) /(U(1) \times U(1))
$$


In this way item $i$ ) of the Theorem 7.1 is proven. It is obvious that the restriction of $p$ on the non-trivial factor gives the map we are needed in order to prove $i$ ). Finally, item $i i i$ ) is a trivial statement and what else has to be done is to compute the reduced symplectic form. In the non-homogeneous coordinates

$$
\left(\zeta_{1}, \zeta_{2}\right)=\left(z_{2} / z_{1}, z_{4} / z_{3}\right)
$$

over $P^{1} \times P^{1}, \quad p$ is simply

$$
p\left(z_{1}, z_{2}, z_{3}, z_{4}\right)=\left(\zeta_{1}, \zeta_{2}\right) .
$$

Referring to Lemma 7.2 we can write

$$
p^{*} \Omega_{\mu}(E)=\Omega_{\mid S^{3} \times S^{3}},
$$

where $S^{3} \times S^{3}$ are spheres defined above. An easy check in coordinates shows that this is true which means that Theorem 7.1 is also proved.

Definition 7.3 The line bundle L over the compact Kähler manifold $X$ is called positive, if

$$
\int_{\sigma} c_{1}(L) \geq 0, \quad \text { for every positively oriented cycle } \sigma \in H_{2}(X, \mathbb{Z}) .
$$

For this type of bundles $H^{0}(X, \mathcal{O}(L)) \neq 0$.

Theorem 7.4 (see Griffiths $\&$ Harris [24]). The group $H^{2}\left(P^{1} \times P^{1}\right)=\mathbb{Z} \oplus \mathbb{Z}$ is generated by $\left[\omega_{1}\right],\left[\omega_{2}\right]$ and

$$
c_{1}\left(N_{F}^{1 / 2}\right)=-\frac{1}{2} c_{1}\left(P^{1} \times P^{1}\right)=-\left(\left[\omega_{1}\right]+\left[\omega_{2}\right]\right) .
$$

In view of the prequantization condition (2.2) we have

$$
\frac{1}{2 \pi} \Omega_{\mu}(E)=N_{1} \omega_{1}+N_{2} \omega_{2}, \quad N_{1}, N_{2} \in \mathbb{Z},
$$

which means that

$$
\begin{gathered}
2 \alpha+\lambda \mu=\lambda N_{1} \\
2 \alpha-\lambda \mu=\lambda N_{2},
\end{gathered}
$$

as well

$$
\mu=\frac{1}{2}\left(N_{1}-N_{2}\right), \quad \lambda=4 \alpha\left(N_{1}+N_{2}\right) .
$$


Introducing $N=\frac{1}{2}\left(N_{1}+N_{2}\right)$, we get immediately $N_{1}=N+\mu, N_{1}=N-\mu$ as well the energy spectrum of the MIC-Kepler problem $E_{N}=-\alpha^{2} / 2 N^{2}$. The Hilbert space $H^{0}\left(P^{1} \times P^{1}, Q_{N}\right)$ is non-trivial if the first Chern class of the line bundle $Q_{N} \rightarrow P^{1} \times P^{1}$

$$
c_{1}\left(Q_{N}\right)=\left(N_{1}-1\right)\left[\omega_{1}\right]+\left(N_{2}-1\right)\left[\omega_{2}\right]
$$

is positive, i.e. $N_{1}, N_{2} \geq 1$ and $N \geq|\mu|+1$. Finally, the degeneracies $m\left(E_{N}\right)$ of the energy levels $E_{N}$ which coincide with dimensionalities of the spaces of holomorphic sections of quantum line bundles $Q_{N}$ are calculated by Riemann-Roch-Hirzebruch theorem:

$$
m\left(E_{N}\right)=\operatorname{dim} H^{0}\left(\mathcal{O}_{\mu}(E), Q_{N}\right)=N_{1} N_{2}=N^{2}-\mu^{2} .
$$

Remark 7.5 The observables $M_{3}$ and $A_{3}$ in the complete set which survive under reduction can be expressed in the nonhomogeneous coordinates $\left(\zeta_{1}, \zeta_{2}\right)$ over $\mathcal{O}_{\mu}\left(E_{N}\right)$ as follows:

$$
\begin{aligned}
& M_{3}^{\mu, N}=\frac{N_{1}}{2} \frac{1-\left|\zeta_{1}\right|^{2}}{1+\left|\zeta_{1}\right|^{2}}-\frac{N_{2}}{2} \frac{1-\left|\zeta_{2}\right|^{2}}{1+\left|\zeta_{2}\right|^{2}} \\
& A_{3}^{\mu, N}=\frac{N_{1}}{2} \frac{1-\left|\zeta_{1}\right|^{2}}{1+\left|\zeta_{1}\right|^{2}}+\frac{N_{2}}{2} \frac{1-\left|\zeta_{2}\right|^{2}}{1+\left|\zeta_{2}\right|^{2}} .
\end{aligned}
$$

The expression for the third component of the Runge-Lenz vector is actually the momentum mapping of the circular action around vertical axes of the spheres. If we fix its value to be $\ell$ then the momentum manifold

$$
N_{1} \frac{\left|\zeta_{1}\right|^{2}}{1+\left|\zeta_{1}\right|^{2}}+N_{2} \frac{\left|\zeta_{2}\right|^{2}}{1+\left|\zeta_{2}\right|^{2}}=\frac{N_{1}}{2}+\frac{N_{2}}{2}-\ell=N-\ell
$$

is either the sphere $S^{3}$ when $N-\ell>0$, four points when $N-\ell=0$ or the empty set in the case $N-\ell<0$. This can be seen quite easily if we introduce the following set of coordinates

$$
\xi_{1}=\left(\frac{N_{1}}{1+\left|\zeta_{1}\right|^{2}}\right)^{1 / 2} \zeta_{1}, \quad \xi_{2}=\left(\frac{N_{2}}{1+\left|\zeta_{2}\right|^{2}}\right)^{1 / 2} \zeta_{2}
$$

in which (7.3) becomes obviously

$$
\left|\xi_{1}\right|^{2}+\left|\xi_{2}\right|^{2}=N-\ell
$$

In the first of the above listed cases we have a free action of $S O(2)$ on $J^{-1}(\ell)$ and therefore we can factorize it. The reduced manifold is topologically the sphere $S^{2}$ and the reduced symplectic form is

$$
\omega_{\ell}=2 \pi(N-\ell) \sigma
$$

where $\sigma$ is the form (7.2) written in any of the non-homogeneous coordinates on the projective line $\left[\xi_{1}: \xi_{2}\right]$. Now the quantization condition reads

$$
(N-\ell) \sigma-\sigma=k \sigma, \quad k \geq 0
$$


from which follows that the maximal value of $\ell$ is $N-1$. Using Riemann-Roch theorem one can easily find that the number of the global holomorphic sections of the reduced quantum bundle $L_{k}$ over the sphere $S^{2}$ is $k+1=N-\ell$. Introducing $\xi:=\xi_{1} / \xi_{2}$ the last function $M_{3}^{\mu, N}$ from the complete set of observables can be written as

$$
(N-\ell) \frac{1-|\xi|^{2}}{1+|\xi|^{2}}+\mu
$$

while the corresponding "quantum" operator is

$$
-2 \xi \frac{\partial}{\partial \xi}+N-\ell-1+\mu
$$

The spectrum of this operator in $\Gamma\left(S^{2}, \mathcal{O}\left(L_{k}\right)\right)$ consists of equidistant of step two integer or half-integer eigenvalues $m \in[-k+\mu,-k+\mu+2, \ldots,-k+\mu-2, k+\mu]$. At classical level (7.8) is just the momentum map of the circle action around the third axe of the sphere $S^{2}$ (so we can forget the additive constant $\mu$ ) and if $|m|<N-\ell$ this action is free. This means that the inverse image of the momentum map is a circle and after reduction we end with a point as reduced phase space. The representation space associated with this point is one-dimensional as the only $S O(2)$-invariant section which descends from $S^{2}$ is the constant section. This can be seen also in another way if we remember that since the very first days of quantum mechanics there are attempts to associate the volume in the phase space with the number of the pure states. This was proven to be assymptocally true (up to universal factor) by Heckman on the basis of the Duistermaat-Heckman exact stationary phase formula [25]. The main ingredient of this formula is the set of fixed points of the action which till now were not taken into account in our considerations. The $S^{1}$ action on $S^{2}$ was treated in [26] and the result (in our notation) is :

$$
\operatorname{vol}\left(S_{m}^{2}\right)=\left\{\begin{array}{lll}
1 & \text { if }|m|<N-\ell, & \text { a point } \\
0 & \text { if }|m| \geq N-\ell, & \text { empty. }
\end{array}\right.
$$

The $S^{1}$ diagonal action on $S^{2} \times S^{2}$ which has four fixed points mentioned above is studied by $\mathrm{Wu}[27]$ and in that case

$$
\operatorname{vol}\left(\left(S^{2} \times S^{2}\right)_{\ell}\right)= \begin{cases}2 \pi(N-\ell) & \text { if } N-\ell>0, \\ 0 & \text { if } N-\ell \leq 0 .\end{cases}
$$

Finally the volume of the orbit manifold $\mathcal{O}_{\mu}(N)$ is $4 \pi^{2} N_{1} N_{2}$ and all this coincides with the results we have obtained before.

In view of the complete coherence of the results obtained at all level of consideration, starting with the extended and ending with a point, we can conclude that the reductionquantization technique is the right and straightforward formalism for the treatment of systems with high symmetries. The missing quantum numbers can be derived by quantizing various symplectic manifolds which appear at different stages of the reduction procedure. 


\section{References}

[1] P. Dirac, Quantum mechanics and a preliminary investigation of the hydrogen atom, Proc. Roy. Soc. A, 110 (1926) 561-579

[2] I. E. Segal, Quantization of nonlinear systems, J. Math. Phys. 1 (1960) 468-488

[3] B. Kostant, Quantization and unitary representations, Lect. Notes Math., vol. 170 (1970) 87-208

[4] J.-M. Souriau, Structure des Systemes Dynamics, Dunod, Paris, 1970

[5] D. Simms and N. Woodhouse, Lectures on Geometric Quantization, Lect. Notes Phys., vol. 53, Springer, New York, 1976

[6] P. Robinson and R. Rawnsley, The metaplectic representations, $M p^{c}$ structures and geometric quantization, Memoirs AMS vol.410, 1989

[7] J. Sniatycki, Geometric Quantization and Quantum Mechanics, Springer, Berlin, 1980

[8] J. Czyz, On Geometric quantization and its connections with the Maslov theory, Rep. Math. Phys., 15(1979) 57-97

[9] H. Hess, On geometric quantization scheme generalizing those of Kostant-Souriau and Czyz, Lect. Notes Phys. vol. 139 (1981) 1-35

[10] J. Marsden and A. Weinstein, Reduction of symplectic manifolds with symmetry, Rep. Math. Phys., 5 (1974) 121-130

[11] M. Kummer, On the construction of reduced phase space of a Hamiltonian system with symmetry, Indiana Univ. Math. J., 30 (1981) 281-291

[12] J. Sniatycki, Constraints and quantization, Lect. Notes Math., vol. 1037 (1983) 301334

[13] V. Guillemin and S. Sternberg, Geometric quantization and multiplicities of group representations, Invent. math., 67 (1982) 515-538

[14] M. Puta, On the reduced phase space of a cotangent bundle, Lett. Math. Phys, 8 (1986) 189-194

[15] M. Gotay, Constraints, reduction and quantization, J. Math. Phys., 27 (1986) 20512066

[16] H. McIntosh and A. Cisneros, Degeneracy in the presence of a magnetic monopole J. Math. Phys., 11 (1970) 896-916 
[17] I. Mladenov and V. Tsanov, Geometric quantization of the MIC-Kepler problem, J.Physics A : Math. \& Gen., 20 (1987) 5865-5871

[18] D. Simms, Geometric quantization of the energy levels in the Kepler problem, Symposia Mathematica, vol XIV (1974) 125-137

[19] I. Mladenov and V. Tsanov, Geometric quantization of the multidimensional Kepler problem , J. Geom. Phys., 2 (1985) 17-24

[20] T. Iwai and Y. Uwano, The four-dimensional conformal Kepler problem reduces to three-dimensional Kepler problem with a centrifugal potential and Dirac monopole field. Classical theory, J. Math. Phys., 27 (1986) 1523-1529

[21] I. Mladenov, Geometric quantization of the MIC-Kepler problem via extension of the phase space, Annales de l'Institute Henri Poincare, 50 (1989) 219-227

[22] V. Guillemin and S. Sternberg, Variations on the theme by Kepler, AMS Colloquium Publications, vol. 42, AMS, Providence, RI, 1990

[23] M. Audin, The Topology of the Torus Actions on Symplectic Manifolds, Birkhäuser, 1991

[24] Ph. Griffiths and J.Harris, Principles of Algebraic Geometry, Wiley, New York, 1978

[25] J. Duistermaat and G. Heckman, On the variation in the cohomology of the symplectic form of the reduced phase space, Invent. math., 69 (1982) 259-268 ; Addendum, ibid. 72 (1983) 153-158

[26] E. Witten, Two dimensional gauge theories revisited, J. Geom.\& Phys. 9 (1992) 303-368

[27] S. Wu, An integration formula for the square of moment maps of circle actions, Lett. Math. Phys. 29 (1993) 311-328 